\title{
LOCAL INHIBITION OF ANGIOGENESIS RESULTS IN AN ATROPHIC NON-UNION IN A RAT OSTEOTOMY MODEL
}

\author{
M. Fassbender ${ }^{1,2^{*}}$, C. Strobel ${ }^{1,2}$, J.S. Rauhe ${ }^{1,2}$, C. Bergmann ${ }^{1,2}$, G. Schmidmaier ${ }^{3}$ and B. Wildemann ${ }^{1,2}$ \\ ${ }^{1}$ Julius Wolff Institute, Center for Musculoskeletal Surgery, Charité-Universitätsmedizin Berlin, Germany \\ ${ }^{2}$ Berlin-Brandenburg Center for Regenerative Therapies, Charité-Universitätsmedizin Berlin, Germany \\ ${ }^{3}$ Department Orthopädie, Unfallchirurgie und Paraplegiologie, Universitätsklinikum Heidelberg, Germany
}

\begin{abstract}
Long bone and in particular tibia fractures frequently fail to heal. A disturbed revascularisation is supposed to be a major cause for impaired bone healing or the development of non-unions. We aim to establish an animal model, which reliably mimics the clinical situation. Human microvascular endothelial cells (HMEC-1) and primary human osteoblast like cells (POBs) were cultured with different angiogenesis-inhibitors (Fumagillin, SU5416, Artesunate and 3,5,4'-Trimethoxystilbene) released out of poly(D,L-Lactide) (PDLLA) coated k-wires and cell activity was determined. Discs containing PDLLA or PDLLA + Fumagillin/Artesunate were placed at the chorionallantoic membrane of hen eggs and the effect on vessel formation and egg vitality was observed. Tibia osteotomy was performed in rats and stabilised with K-wires coated with PDLLA + Fumagillin or with PDLLA only (control group). The healing was compared at different time points to the PDLLA control. Fumagillin and Artesunate inhibited the activity of HMEC-1 with minor effect on POBs. Artesunate caused embryonic death, whereas Fumagillin had no effects on egg vitality, but reduced the blood vessels. In the animal study all rats showed an impaired healing with reduced biomechanical stability. The Fumagillin treated tibiae had a significantly decreased radiographic callus size at day 42 and 84, less blood vessels in the early callus, a reduced histological callus size at day 10, 28 and 84, as well as an altered callus composition. This study presents a less vascularised, atrophic, tibia non-union and can be used in further investigations to analyse the pathology of atrophic non-union and to test new interventions.
\end{abstract}

Keywords: bone healing, angiogenesis inhibition, atrophic non-union

*Address for correspondence:

M. Fassbender

Berlin-Brandenburg Centre for Regenerative Therapies

Charité-Universitätsmedizin

Föhrerstrasse 15, 13353 Berlin, Germany

E-mail: mirja.fassbender@charite.de

\section{Introduction}

Despite recent improvements in surgical methods, fracture healing, especially of long bones, is still accompanied by severe complications. Tibia fractures are reportedly the most common long bone lesions and have the highest non-union rate ranging from $1 \%$ to $80 \%$ depending on the anatomical localisation and the severity of the trauma (Tzioupis and Giannoudis, 2007). Weber described a scoring system, which is based on the radiographic appraisal of non-unions (Weber, 1982). According to this classification, non-unions are separated in different vital and avital forms. Hypertrophic non-unions are considered to be biologically active and vascularised whereas atrophic non-unions are considered to be relatively biologically inactive and more or less avascular. But, patients with nonunions are rarely easily compared and it is now known, that more precise classification systems with respect to the individual situation of the patient are needed (Calori et al., 2008). Contradictory findings about the vascularity at the lesion site in case of healing failure had been made. Significantly higher non-union rate and a greater number of weeks to union were observed in fractures associated with an injury to the posterior tibial artery than in fractures without this vascular injury (Brinker and Bailey, 1997). In contrast to this a further study was not able to confirm a difference in vessel density comparing biopsies taken from the fracture gap of patients who developed a hypertrophic or an atrophic non-union or from patients who showed a regular healing pattern (Reed et al., 2002). Despite this debate about the definition of non-unions, it is currently assured that vascularisation plays an important role in fracture healing interactions (Giannoudis et al., 2008). Both pathways, the angiogenic and the osteogenic, are intimately linked and are sharing molecular key factors e.g. the vascular endothelial growth factor or the bone morphogenetic proteins (Deckers et al. 2002; Dai et al., 2007; Beamer et al., 2009). But, it is not understood why some patients do develop a non-union and some patients do not and which endogenous factors are miss-regulated in these patients. Inhibitors of angiogenesis are clinically used in cancer therapy and other diseases (Folkman, 2004; Sivakumar et al., 2004; Folkman, 2007). One of the most potent antiangiogenic agents is Fumagillin, a naturally secreted antibiotic of the fungus Aspergillus fumigatus (Ingber et al., 1990). Fumagillin inhibits the methionineaminopeptidase type II through covalent binding (Sin et al., 1997; Liu et al., 1998), which leads more downstream to a block of the noncanonical Wnt-signalling pathway (Zhang et al., 2006). SU5416 [3-(3,5-dimethyl-1H-pyrrol2-ylmethylene)-1,3-dihydro-indol-2-one] is a selective 
inhibitor of the vascular endothelial growth factor (VEGF)receptor 2 tyrosine kinase. It inhibits VEGF-dependent endothelial cell proliferation in vitro and in vivo (Ye et al., 2006) and was the first VEGF-receptor 2 inhibitor to enter clinical development. Artesunate, a derivative of Artemisinin, has a long tradition in the treatment of fever and malaria (Klayman, 1985), but also possess antitumor activity and antiangiogenic potential (Efferth et al., 2003; Chen et al., 2004; Dell'Eva et al., 2004) by lowering VEGF and VEGF-receptor expression (Chen et al., 2004; Zhou et al., 2007). 3,5,4'-Trimethoxystilbene is a herbal antioxidant present in high concentrations in red wine (Frémont, 2000; Belleri et al., 2005). This factor acts through down regulation of VEGF-receptor 2 and cellcycle modulation (Alex et al., 2010) causing microtubule disorganization in vitro as well as inhibition of blood vessel growth in ovo and in vivo (Belleri et al., 2005). A disturbed revascularisation after bone lesion has been so far created in different animal models by intensive mechanical interference, e.g. destruction of the periosteum, endosteum or the medullary canal or a combination of these techniques (Triffit et al., 1993; Hietaniemi et al., 1995; Brownlow et al., 2002; Reed et al., 2003) or an induction of a unilateral total hind limb ischemia (Lu et al., 2007). One study applied systemically a synthetic Fumagillin analogue (TNP-470) in a rat femur fracture model, but they did not analyse the effect on revascularisation (Hausman et al., 2001). Until there is no causal clinical therapy for the treatment of nonunions available, reliable animal models, which mimic the clinical situation and investigate the role of blood supply after bone trauma, are essential. In this study we aimed firstly to select a substance which inhibits selectively endothelial proliferation without having an impact on bone forming cells. For this purpose in vitro experiments were performed to test the influences of Fumagillin, SU5416, Artesunate and 3,5,4' -Trimethoxystilbene on endothelial cells and osteoblast like cells and on the vascularisation in an in-ovo model. Further on, the selected substance was tested in vivo after tibia osteotomy. For this purpose we used an impaired healing model, which had already been established by our group (Kratzel et al., 2008) as control. Bone healing parameters were compared between the PDLLA control group (tibia osteotomy and stabilisation with inhibitor-free PDLLA coated wires) and the treatment group (tibia osteotomy and stabilisation with PDLLA/ inhibitor coated wires).

\section{Material and Methods}

\section{Polymer coating of titanium kirschner-wires (k-wires)}

We used the polymer poly(D,L-Lactide) (PDLLA) (Boehringer Ingelheim, Ingelheim, Germany), for incorporating substances. The release of factors from this coating showed an initial burst release within the first 2 days followed by a sustained release over the following 40 days (Schmidmaier et al., 2001). PDLLA was dissolved in ethyl acetate $(100 \mathrm{mg}$ in $1.5 \mathrm{ml}$ ) (Sigma-Aldrich, München, Germany) and the same absolute amounts for the angiogenesis inhibitors Fumagillin (Enzo Life Science, Lörrach, Germany), SU5416 (Sigma Aldrich,
München, Germany), Artesunate (Sigma Aldrich), and 3,5,4'Trimethoxystilbene (Biomol GmbH, Hamburg, Germany) were incorporated in the polymer solution at $5 \%(3 \mu \mathrm{g}), 10 \% \mathrm{w} / \mathrm{w}(6 \mu \mathrm{g})$ and $20 \% \mathrm{w} / \mathrm{w}(12 \mathrm{~g})$ weight per weight $(\mathrm{w} / \mathrm{w})$ PDLLA. The k-wires $(\varnothing 1 \mathrm{~mm}$; Synthes, Oberdorf, Switzerland) were coated under sterile conditions by dipping twice and drying immediately by vaporisation of the solvent. The coated wires were stored sterile packed at $-20{ }^{\circ} \mathrm{C}$ with desiccant. All steps of the coating were performed under sterile conditions.

\section{Cell culture and assessment of metabolic activity}

For in vitro experiments human microvascular endothelial cells (HMEC-1) and primary human osteoblast like cells (POBs) were treated with 4 different angiogenesis inhibitors released from PDLLA coated wires. The HMEC-1 cell line was established by Ades et al. (1992) and was kindly provided by Prof. G. Schönfelder (Institut für Klinische Pharmakologie und Toxikologie, CharitéUniversitätsmedizin Berlin, Germany). The POBs were obtained from three different donors with their written consent. For cell isolation, bone was harvested during knee joint replacement and the trabecular bone was minced into little pieces followed by overnight digestion with collagenase Type II (Biochrom, Berlin, Germany) according to established protocols (Robey and Termine, 1985). The cells were cultured at $37^{\circ} \mathrm{C}, 5 \% \mathrm{CO}_{2}$, and $95 \%$ humidity in MEME/F12 1:1 + $10 \%$ foetal calf serum $(\mathrm{FCS})+1 \%$ penicillin/streptomycin $(\mathrm{PS}),+0.05$ $\mathrm{mM}$ L-ascorbate-2-Phosphate, + $0.05 \mathrm{mM}$ ß-glycerolPhosphate (Biochrom) (POB), or MCDB 131 media (Invitrogen, Darmstadt, Germany) + $2 \mathrm{mM}$ L-Glutamine, $5 \%$ FCS, $1 \%$ PS (Biochrom) + freshly supplemented $1 \mu \mathrm{g} / \mathrm{mL}$ hydrocortisone (Sigma-Aldrich) (HMEC-1), respectively.

We seeded the cells in 96-well plates $\left(5 \times 10^{3}\right.$ vital cells/ well/100 $\mu \mathrm{L}$ of HMEC-1 and $1 \times 10^{3}$ vital cells/well/100 $\mu \mathrm{L}$ of POBs). After $3 \mathrm{~h}$ (HMEC-1) or overnight incubation (POBs), Alamar Blue (Biozol, Eching, Germany) (1:10 in media) was added to measure metabolic cell activity. Optical density was measured after $3 \mathrm{~h}$ incubation (Microplate Reader; Bio-Rad Laboratories, München, Germany). Subsequently, coated kirschner-wires were added to the cells with fresh media and continued culturing. We repeated the Alamar Blue Assay after 3 and $6 \mathrm{~d}$. The experiments were performed in quadruplicates and repeated twice.

\section{Hen's egg chorioallantoic membrane test (HET-CAM Test)}

Fertilised domestic chick embryos were incubated at $37{ }^{\circ} \mathrm{C}, 70 \%$ humidity and periodic rotation. On day 6 a small hole was formed by carefully removing the shell membrane and the chorioallantoic membrane was photographed. A small round filter paper (approximately $5 \mathrm{~mm}$ in diameter) containing PDLLA and PDLLA with incorporated inhibitors Fumagillin or Artesunate (10 $\% \mathrm{w} / \mathrm{w}$ ) was gently placed on the exposed area of the chorioallantoic membrane. The incubation was continued with the window upright for $30 \mathrm{~h}$. After removing the eggs from the incubator, the chorioallantoic membrane 
Table 1: Investigated healing parameters of the osteotomised tibia at the different postoperative time points.

\begin{tabular}{|c|c|c|c|c|c|}
\hline \multirow{2}{*}{ group/day } & \multicolumn{4}{|c|}{ Time points (day) and performed methods } & \multirow{2}{*}{$\begin{array}{l}\text { number } \\
\text { of rats }\end{array}$} \\
\hline & $0,28,42,84$ & 5,10 & $28,42,84$ & 42,84 & \\
\hline $\begin{array}{l}\text { PDLLA control } \\
\text { ( } 6^{*} / \mathrm{meth} \text { od } / \text { time } \\
\text { point })\end{array}$ & \multirow{2}{*}{$\begin{array}{l}\text { in vivo } \\
\text { radiology }\end{array}$} & \multirow{2}{*}{$\begin{array}{c}\text { vessel } \\
\text { density, } \\
\text { callus size }\end{array}$} & \multirow{2}{*}{$\begin{array}{c}\text { histology/ } \\
\text { histomorphometry }\end{array}$} & \multirow{2}{*}{$\begin{array}{l}\text { biomechanical } \\
\text { testing }\end{array}$} & 42 \\
\hline $\begin{array}{l}\text { Fumagillin } \\
\text { ( } 8^{*} / \mathrm{meth} \text { od/time } \\
\text { point) }\end{array}$ & & & & & 56 \\
\hline
\end{tabular}

* except for biomechanical testing: $n=5$ (PDDLA control, 84 days) and $n=7$ (Fumagillin, 42 and 84 days).

was photographed a second time. Egg vitality and blood vessel appearance was evaluated visually by three different observers regarding vessel formation.

\section{Surgical model}

All animal experiments were approved by the local authorities. Five month old female Sprague Dawley rats, weighing 250-280 g, were used. A detailed description of the surgery and some results of the PDLLA control group have already been published (Kratzel et al., 2008). Briefly, the medullar cavity of the tibia was opened and reamed twice. The tibia was osteotomised at the midshaft level using a diamond disk (HORICO, Berlin, Germany). For stabilisation a kirschner-wire coated with substance-free PDLLA (control) or $10 \% \mathrm{w} / \mathrm{w}$ Fumagillin in PDLLA was inserted from the proximal end of the tibia into the medullar canal. The fibula was fractured manually. The wound was closed and gentamycin ointment was applied locally. Euthanasia was performed in deep anaesthesia by an intracranial injection of potassium chloride.

The former PDLLA control group was not re-operated, because we followed the 3-R principle (replace, reduce and refine) for animal testing, but the animals were operated by the same surgeon within 12 month. Healing outcomes were followed as stated in Table 1.

\section{Radiographic analysis}

Radiographs were taken (46 KV/2.5 mAS; Kodak DirectView CR Cassette; Mobilett Plus X-ray unit Siemens AG, Erlangen, Germany) (posterior-anterior / lateromedial) after surgery and at day 5, 10, 28, 42 and 84 (Table 1). The callus diameter proximally and distally adjacent to the osteotomy gap was analysed by one observer (MF) using Image-J Version 1.30 (National Institutes of Health, Bethesda, MD, USA). Each measurement was performed in both views and repeated three times. The mean coefficient of variance of repeated single measurements was 2.72 $\%$. Mean values were calculated and callus diameter was expressed as a percentage to day 0 .

\section{Biomechanical testing}

We performed biomechanical testing at day 42 and 84 (Table 1) as previously described (Kratzel et al., 2008). For biomechanical testing, the tibiae were fixed in a special device and preloaded with an axial force of 5 Newton. A constant linear propulsion $(1 \mathrm{~mm} / \mathrm{min})$, generated by a material testing machine (Zwick 1455, Ulm, Germany), was applied to a lever arm attached to one of the pivoted axes for transforming the translation of the material-testing machine to a uniform torsional movement. The other side was connected with a load cell $($ Fmax $=50 \mathrm{~N}, \mathrm{HBM}$, Darmstadt, Germany) which recorded the force. Maximum load and torsional stiffness was calculated. The values were expressed as percentages of the contralateral intact tibia.

\section{Histological analysis}

For histological evaluation at days 5, 10, 28, 42 and 84 (Table 1), the soft tissue was removed from the entire tibia taking care not to destroy the callus tissue. Bones were fixed for $48 \mathrm{~h}$ in $10 \%$ normal buffered formalin. Bone specimens taken at day 5 and $10(n=14$ per time point $)$ were decalcified with Ethylenediaminetetraacetate (EDTA), dehydrated, embedded in paraffin and longitudinal sections (4 $\mu \mathrm{m}$; Leica SM 2500s microtome, Wetzlar, Germany) were made. Slices were stained with haematoxylin and eosin (HE). For visualisation of the vessels the paraffin slices were rehydrated, blocked with normal horse serum (Vector Laboratories, Burlingame, CA, USA) and incubated with monoclonal mouse anti human smooth muscle actin (Dako, Glostrup, Denmark; Clone 1A4; dilution 1:100) for $1 \mathrm{~h}$ at $37^{\circ} \mathrm{C}$. Alkaline phosphatase anti-mouse antibody was used as secondary antibody (Vector Laboratories; AP-2000; dilution 1:200; 30 min incubation period at room temperature). Visualisation was done with the alkaline phosphatase substrate kit (Vector Laboratories). Day 28, 42 and 84 ( $n=14$ per time point) samples were transferred after formalin fixation into polymethylmethacrylate (Technovit 9100 neu; Heraeus Kulzer, Wehrheim, Germany). Ten longitudinal sections $(4 \mu \mathrm{m})$ were cut and stained using Safranin-Orange/von Kossa (SafO/vK) and Safranin-Orange/Light green (SafO/ $\mathrm{Lg})$. A region of interest (ROI) was defined, including the zone of reactive callus proximally and distally from the centre of the osteotomy gap extended in length 1.5fold of the individual cortical bone diameter. Within this region, early reactive callus (HE), blood vessel density $(\alpha-$ SMA), amount of mineralised and nonmineralised tissue $(\mathrm{SafO} / \mathrm{vK})$, and cartilaginous tissue $(\mathrm{SafO} / \mathrm{Lg})$ was determined using a DM-RB microscope (Leica) and an image analysing system (KS 400; Zeiss, Göttingen, Germany) as described previously (Schmidmaier et al., 2004; Kratzel et al., 2008). 

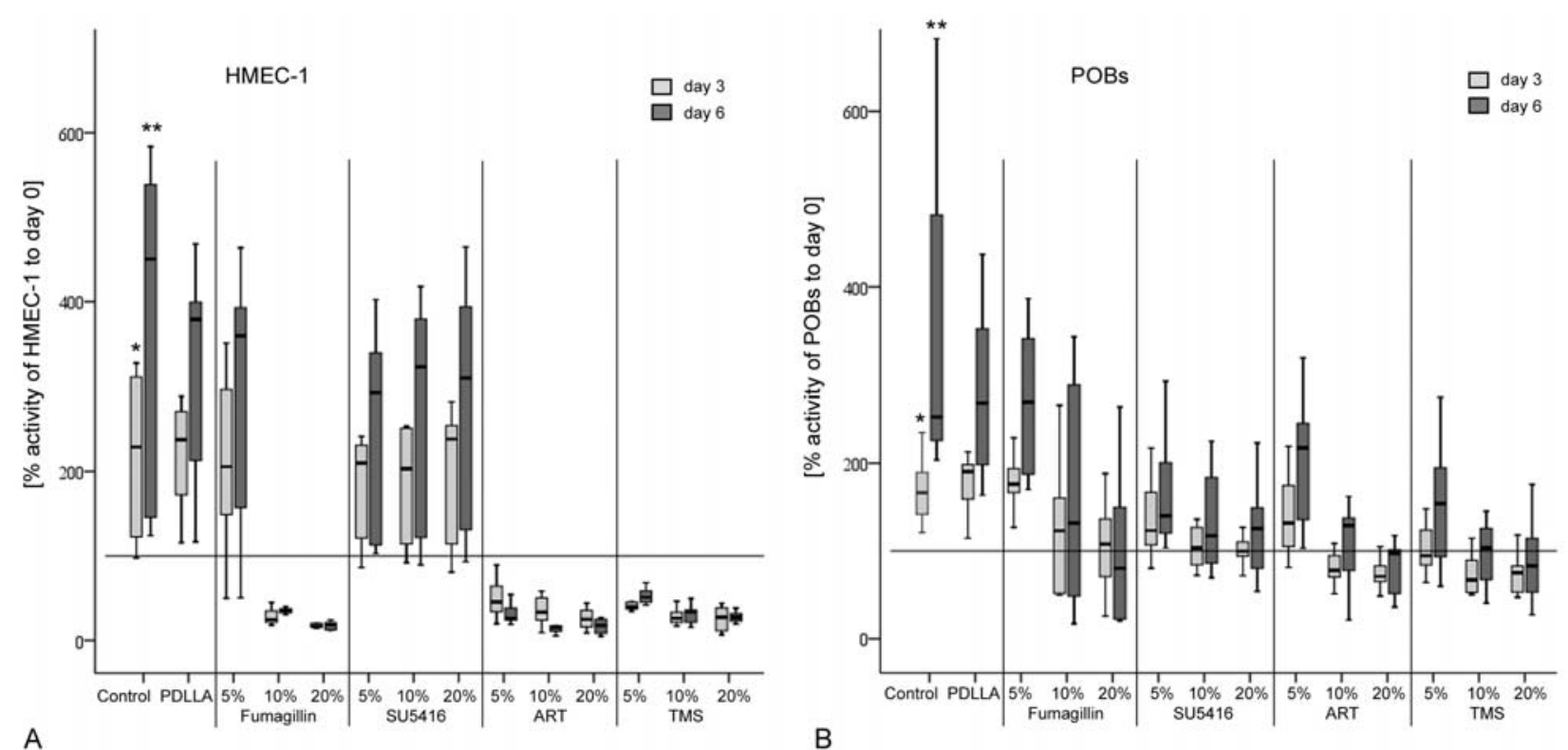

Fig. 1. (A) The diagram shows the metabolic cell activity of human microvascular endothelial cells (HMEC-1). Significant differences due to the drug treatment were seen when compared to the control group (*: day 3; **: day 6). (B) Primary human osteoblast like cells (POBs) at different time points of cultivation (after day $3=$ light gray; after day $6=$ dark gray) with different concentrations of the four different inhibitors (\%w/w in PDLLA) compared to day 0 in percent.

Shown are for both tests the significant differences after Bonferroni-Holm correction. (A) A significant reduction of the metabolic activity of HMEC-1 was achieved by Trimethoxystilbene and Artesunate at 5,10 and $20 \%$ and Fumagillin at $20 \% \mathrm{w} / \mathrm{w}$ of PDLLA (day 3 and $6, p \leq 0.0001$ ) and Fumagillin at $10 \% \mathrm{w} / \mathrm{w}$ of PDLLA (day 3, $p=0.0001$; day 6, $p=0.004$ ). (B) A significant reduction of the metabolic activity of POBs was achieved by Trimethoxystilbene at 5, 10 and $20 \%$, Artesunate at 10 and $20 \%$, SU 5416 at 10 and $20 \%$ (day 3 and $6, p \leq$ 0.007 ), SU 5416 at 5\% (day 6, $p=0.0001$ ) and Fumagillin at $20 \%$ (day 3, $p=0.0005$; day 6, $p=0.0003$ ) and at $10 \% \mathrm{w} / \mathrm{w}$ in PDLLA (day $6, p=0.0029$ ).

\section{Statistical analysis}

Differences in the metabolic activity of HMEC-1 and POBs were related to day 0 and the metabolic activity of HMEC-1 and POBs treated with four different inhibitors at different concentrations was compared to the metabolic activity of untreated cells at both time points. For global comparison of all groups a Kruskal Wallis Test was done $(p<0.001)$. For two group comparison the Mann-Whitney U-Test followed by the Bonferroni Holm method was used. Radiographic callus diameter, vessel formation within the callus, mechanical stability as well as callus size and composition were compared between the Fumagillin treated and PDLLA-treated animals. Again the MannWhitney U-Test was used. All statistical analysis were performed with PASW Statistics 18 (Chicago, IL, USA).

The data listed within the text are presented as median. The data within the figures (boxplots in Figs. 1, 3, 4 and 5) are presented as 50th percentile (median) and 75th percentile (top of each box) and 25th percentile (bottom of each box). The whiskers (the lines that extend out the top and bottom of the box) represented the highest and lowest values that were not outliers or extreme values. In Fig. 6 the median values (including 25th and 75th percentile) of periosteal callus size $\left[\mathrm{mm}^{2}\right]$, as well as the median values (including 25th and 75th percentile) of callus composition (mineralised and cartilage area [\%]) over time are presented.

\section{Results}

\section{In vitro and in ovo effect of angiogenesis inhibitor}

Fumagillin and Artesunate specifically inhibited endothelial cell proliferation with less affecting osteoblasts. The HMEC-1 treatment was associated with a significant inhibiting effect on metabolic cell activity except for Fumagillin at $5 \% \mathrm{w} / \mathrm{w}$ and SU5416 at all concentrations at day 3 and 6 . The POB treatment showed negative effects, too, but for Fumagillin at $5 \% \mathrm{w} / \mathrm{w}$ at day 3 and 6 and at 10 $\% \mathrm{w} / \mathrm{w}$ at day 3 , SU5416 at $5 \% \mathrm{w} / \mathrm{w}$ at day 3 , and Artesunate at $5 \% \mathrm{w} / \mathrm{w}$ at day 3 and 6 differences in cell activity were not significant (Fig. 1). Based on the results of the cell culture experiments $10 \% \mathrm{w} / \mathrm{w}$ of Fumagillin and Artesunate were investigated in the HET-CAM Test. The PDLLA control and Fumagillin had a minor influence on the egg vitality; whereas the application of Artesunate caused rapid embryonic death. The application of Fumagillin resulted in a reduced number of blood vessels and smaller capillaries disappeared after exposure (Fig. 2). Therefore, an implant coating with $10 \%$ Fumagillin was used in the animal experiments.

\section{In vivo effect of Fumagillin on osteotomy healing}

All outcome parameters assessed indicated a delayed healing in both groups and an atrophic non-union with altered callus composition in the Fumagillin treated group 

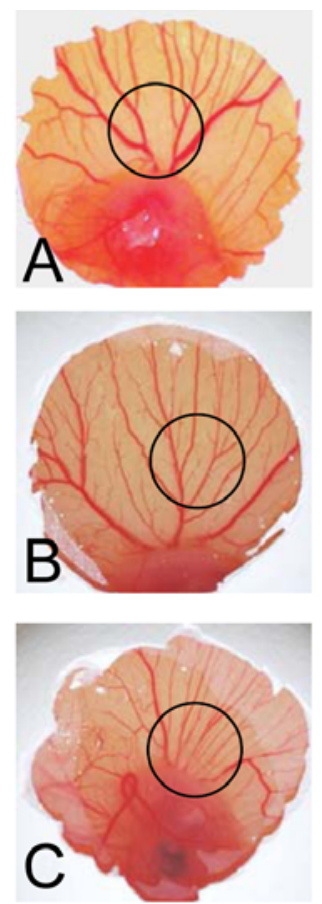

$0 \mathrm{hrs}$
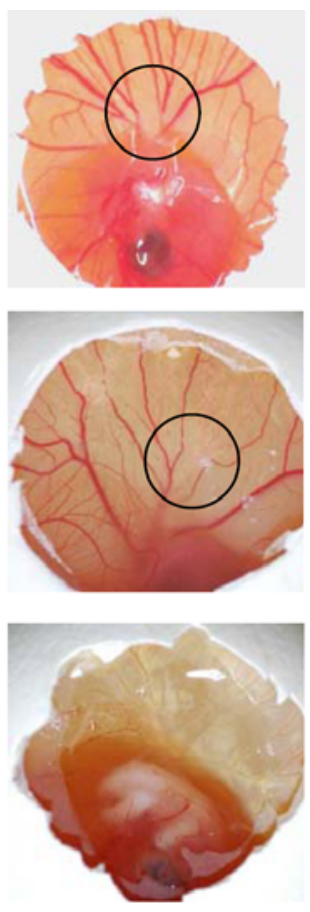

$30 \mathrm{hrs}$

\section{Substance \\ vital eggs}

PDLLA

6

1

6

1

6

Fig. 2. These photographs show exemplarily the results of the HET-CAM Test after filter disc application containing PDLLA (A), Fumagillin (B) and Artesunate (C) respectively the number vital / avital eggs after $30 \mathrm{~h}$ culturing time. The circles within the photographs demonstrated the location of the filter paper disc. PDLLA had no visible effects on blood vessel architecture and in one case out of six an embryonic death was observed. Fumagillin (10 \% w/w in PDLLA) reduced the number of blood vessel, but only one out of six embryos died during the culture. Artesunate $(10 \% \mathrm{w} / \mathrm{w}$ of PDLLA) resulted in avital eggs in all cases.

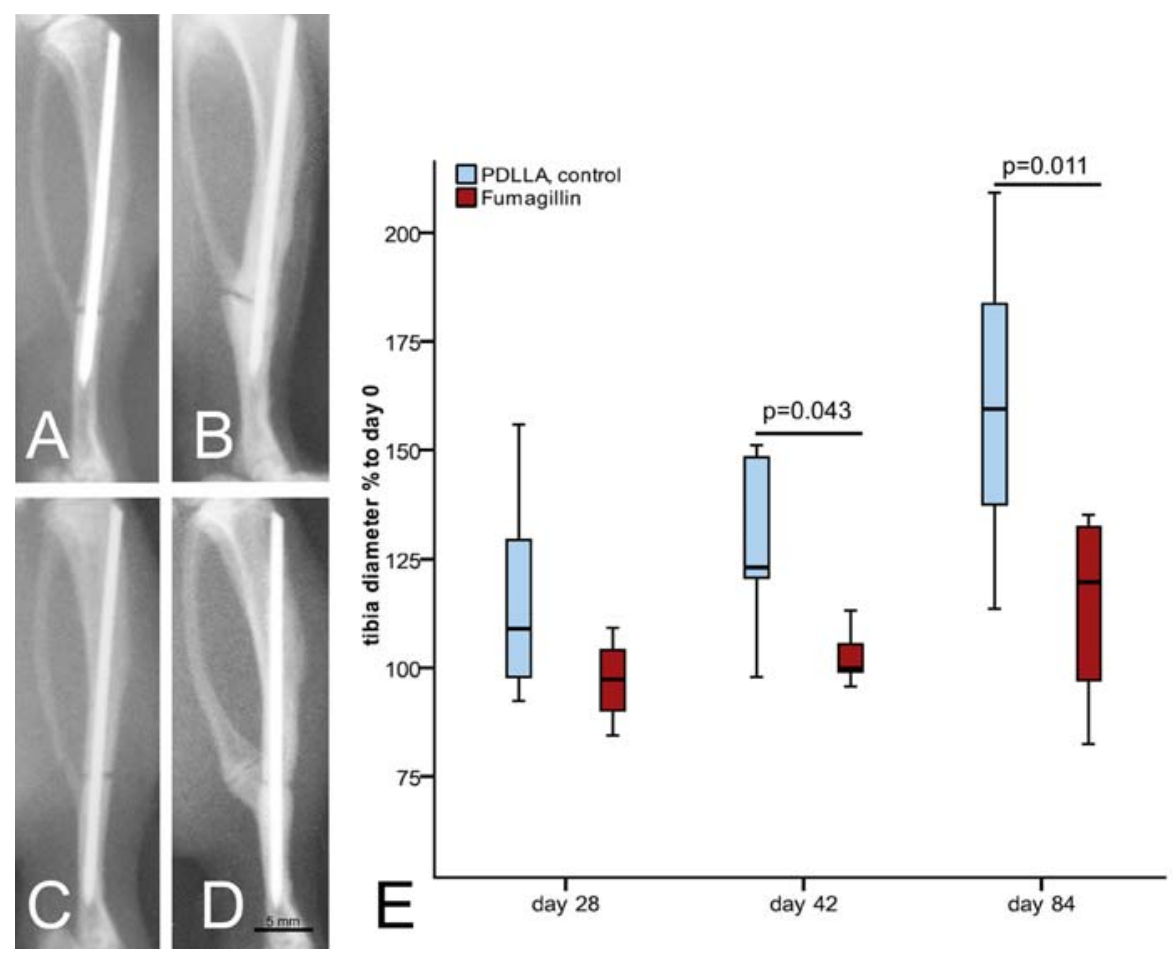

Fig. 3. These images are examples of radiographs of the PDLLA control (A: latero-medial view of day 0, post surgery; B: latero-medial view of day 84) and the Fumagillin group (PDLLA + $10 \%$ w/w Fumagillin) (C: latero-medial view of day 0, post surgery; D: latero-medial view of day 84), scale bar: $5 \mathrm{~mm}$. The latero-medial radiographs showed clearly a hypertrophic non-union in the PDLLA control (B) and less active non-union of the tibia and hypertrophic non-union of the manually fractured and non-stabilised fibula in the Fumagillin group (D). The radiographic evaluation revealed a reduced callus diameter (\% to day 0$)$ in the Fumagillin group at day 42 and at day 84 (E). 


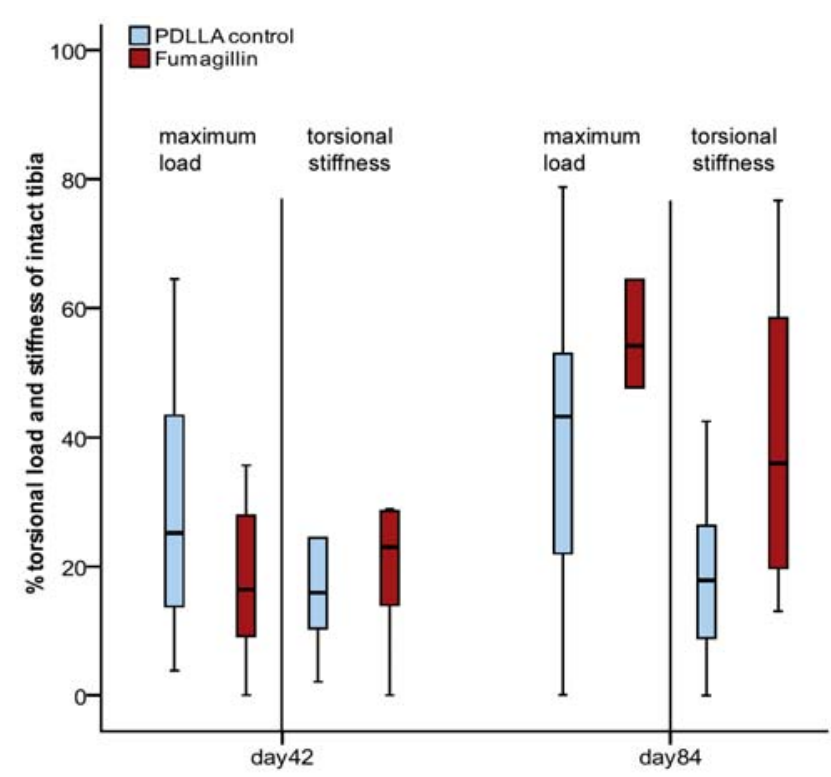

Fig 4. The columns show comparison of the biomechanical properties of the osteotomised tibiae (maximum load and torsional stiffness as \% of contralateral intact tibiae). Number of analysed osteotomised tibiae: $n=6$ (PDDLA control, 42 days), $n=7$ (Fumagillin, 42 days), $n=5$ (PDLLA control, 84 days) and $n=7$ (Fumagillin, 84 days).
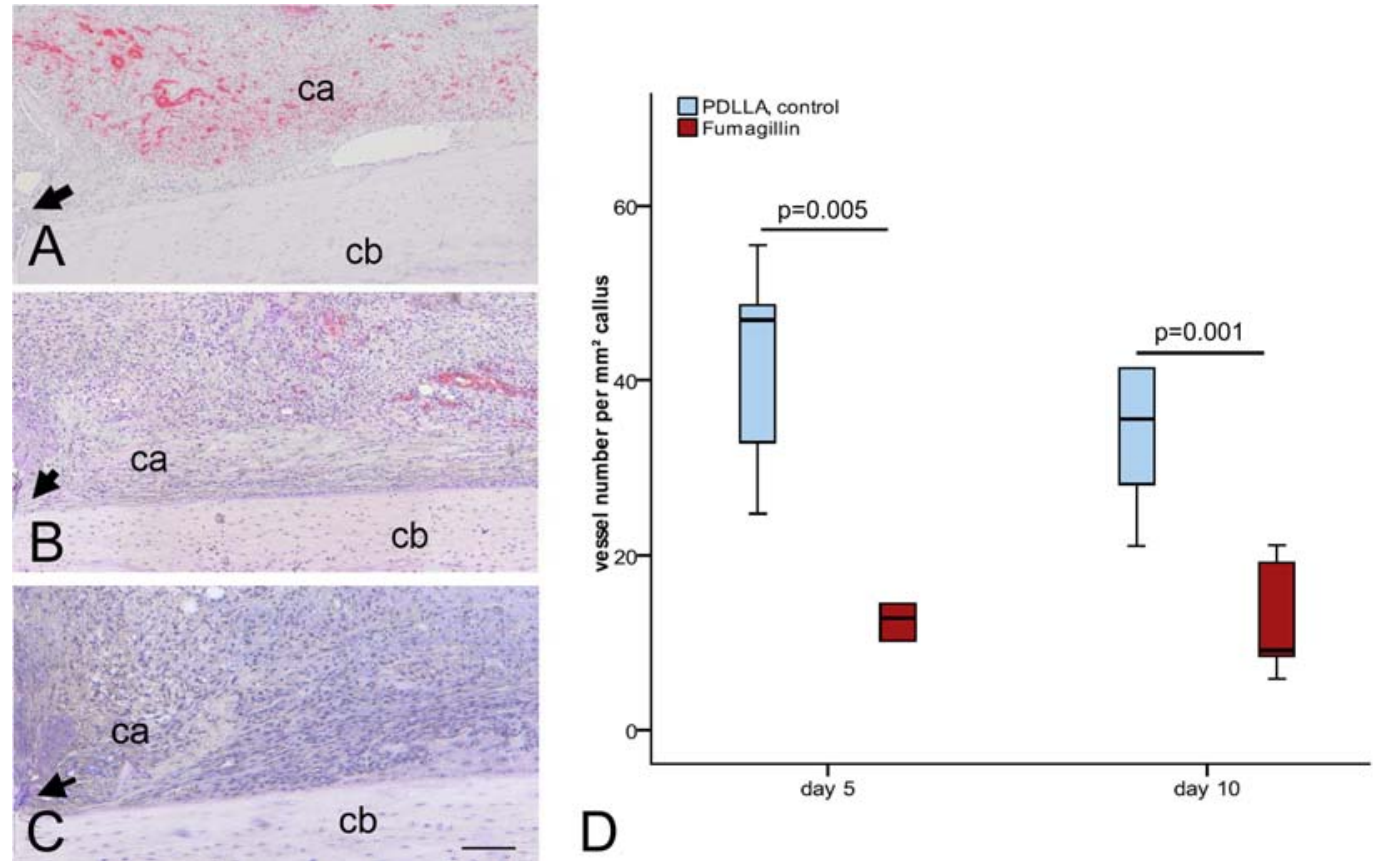

Fig. 5. These images (A, B, C) are examples of blood vessels within the callus at day 5 (A, PDLLA control; $\mathbf{B}$, Fumagillin group; $\mathbf{C}$, negative control, without primary antibody). $\mathrm{cb}=$ cortical bone; $\mathrm{ca}=\mathrm{callus} ; \alpha$-smooth muscle actin (sma)-staining, scale bar: $100 \mu \mathrm{m}$. Vessel density differed significantly between both groups at day 5 and 10 (C).

compared to the untreated PDLLA control group. Beside one animal, which displayed osseous bridging of the tibial cortex in both views, in all other animals the osteotomy gap remained visible until day 84 . The radiographic evaluation revealed a significantly reduced callus diameter in the Fumagillin group at day $42(p=0.043)$ and day $84(p=$ 0.011 ) [\% to day 0] (Fig 3). Maximum load and torsional stiffness showed no significant differences between both groups and in all groups the median values were below
$50 \%$ compared to the intact tibiae, indicating no healing (Fig. 4). The blood vessel counts resulted in a significant reduced vessel density in the Fumagillin group compared to the PDLLA control (Fig. 5) at day $5(p=0.005)$ and day $10(p=0.001)$. In one animal with bone consolidation on the radiograph, bilateral mineralised callus tissue was seen in the histology. All other animals revealed cortical non-union and incomplete periosteal bridging until day 84 with remaining cartilage tissue. Regarding the early 

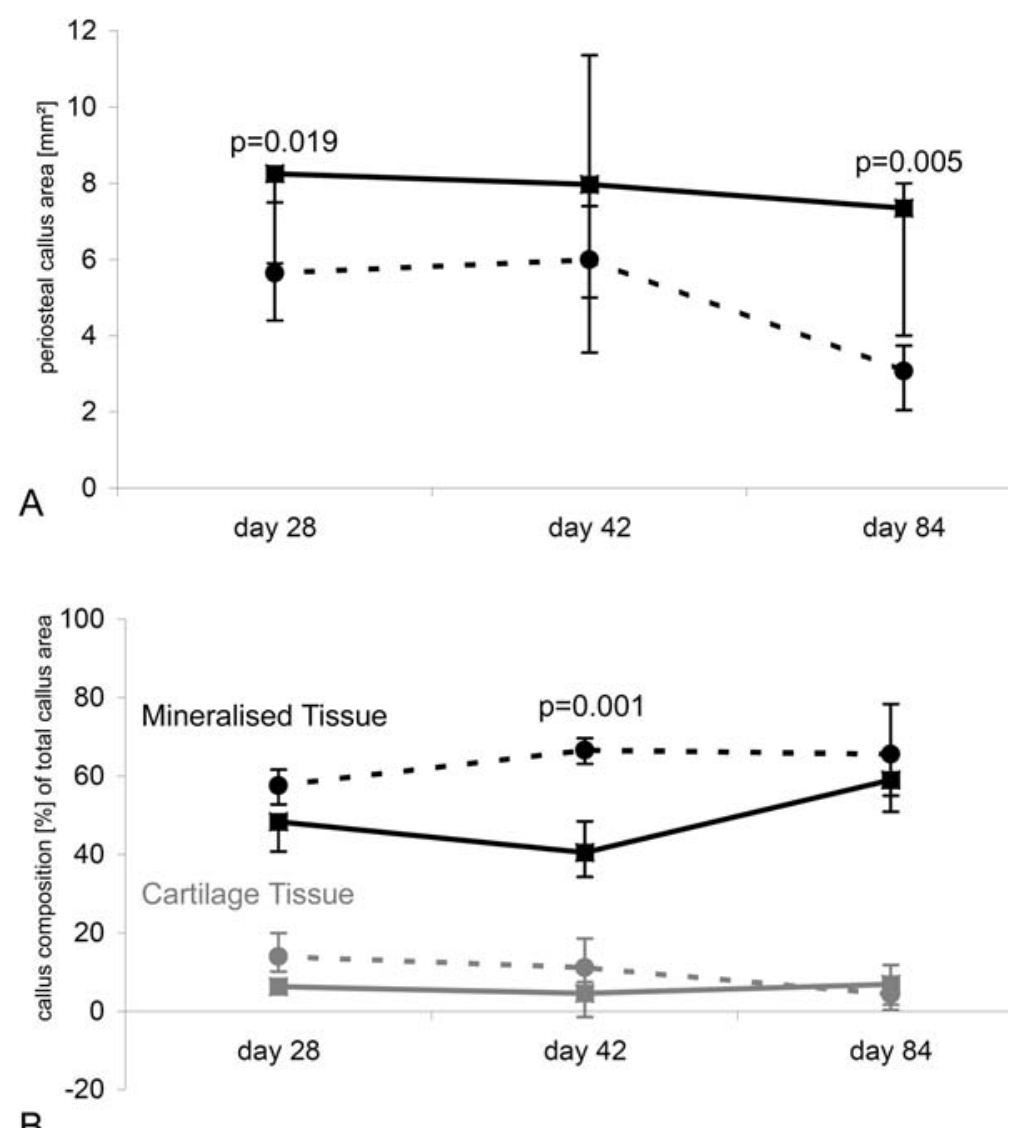

B

Fig. 6. The graphs show histomorphometric results of callus size and callus composition (A, B, continuous line: PDLLA control; dashed line: Fumagillin group; black: mineralised area, gray: cartilage area) within the region of interest. The diagram shows the median and the $25 \%$ and $75 \%$ percentiles. There is significant smaller total periosteal callus in the Fumagillin group at day 28 and day 84, whereas the mineralised tissue fraction was significantly higher in the Fumagillin than in the PDLLA control group at day 42. The cartilage proportion showed nearly similar values.

time points, the evaluation of the total callus size in $\left[\mathrm{mm}^{2}\right]$ showed a reduced callus tissue formation in Fumagillin group compared to PDLLA control at day 5 (9.5 versus 17.7 versus, not significant) and at day 10 (11.2 versus 24.3, $p=0.001)$. Regarding the late time points, the histomorphometry revealed a smaller total periosteal callus $\left(\mathrm{mm}^{2}\right)$ in the Fumagillin group at day $28(p=0.019)$ and day $84(p=0.005)$ (Fig 5.) whereas the mineralised tissue fraction of the callus area $(\%)$ was higher in the Fumagillin than in the PDLLA control at day $42(p=0.001)$ (Fig. 6).

\section{Discussion}

To our knowledge this is the first study to demonstrate, that Fumagillin reduced the number of blood vessels in the early phase of bone healing and therefore inhibited angiogensis when locally delivered from a PDLLA implant coating after bone lesion. This is mainly important with regard to clinical aspects, because a disturbed blood supply is supposed to be a major cause of delayed union or non-union during fracture healing. It has been shown in previous work that the extent of vascular damage is correlated with failure of bone repair (Dickson et al., 1995; Einhorn, 1995; Giannoudis et al., 2008). For the better understanding of the underlying mechanisms and for the development of new therapy options, animal models, which come as close as possible to the clinical situation, are essential. In the present model the defect stabilisation with substance free PDLLA coated wires led to a delayed union with hypertrophic callus appearance, whereas the application of Fumagillin and the disturbance of revascularisation indicated the development of an atrophic non-union, and a reduced callus size and altered callus composition.

To identify a potent angiogenesis inhibitor and the right concentration we performed in vitro and in ovo experiments. The in vitro experiments, aside from SU5416, confirmed the inhibitory action of Fumagillin, Artesunate, and 3,5,4' -Trimethoxystilbene on endothelial cells. A similar dose-dependent negative effect on the proliferation had been described recently in different types of endothelial cells (Wang et al., 2000; Chen et al., 2004; Dell 'Eva et al., 2004; Hou et al., 2009; Alex et al., 2010). The POB-like cells were less affected by adding either antiangiogenic substances. In accordance to that finding a selective mode of action has been proven for Fumagillin during in vitro co-culture of human endothelial and nonendothelial cells (Wang et al., 2000). Based on the fact, that Trimethoxystilbene had a strong inhibiting effect on both cell types, HMEC-1 and POBs, at all concentrations 
and that SU5416 showed no significant inhibiting influence on cell activity of HMEC-1, we continued with Fumagillin and Artesunate for the in ovo HET-CAM Test. Fumagillin not only reduced the formation of new vessels but also seemed to lead to a regression of already existing vessels. This is in accordance with a previously published study reporting a generally reduced vasculature without severe alteration of vessel organisation, a reduced artery and vein length as well as a shrinking of major vein and artery branches (Hazel, 2003). Artesunate caused the death of chicken embryos in the present study. This was also seen by Zhou et al. demonstrating that higher concentrations structurally altered CAMs including cell degeneration and destroyed cell integrity as well infiltration of inflammatory cells (Zhou et al., 2007). This lethal effect of Artesunate seen in the HET-CAM Test, however, will not occur in the adult rats. Based on the very low dosage and the single mode of application we would not expect, that the animals will be severely affected or die after the administration of Artesunate. But, in respect to the high survival rate of about $85 \%$ of the chicken embryos after Fumagillin application, we decided to continue with the Fumagillin for the in vivo experiments.

To our knowledge there is only one study, which used a pharmaceutical approach to inhibit vascularisation during bone healing. Hausman et al. systemically applied the Fumagillin analogue TNP-470 after femur osteotomy in rats (Hausman et al., 2001). In contrast to this, in the current study the antiangiogenic substance has been incorporated in the PDLLA-coating of k-wires, which were used for mechanical stabilisation. Local delivery patterns can achieve high concentrations of the active substances at the interface of implant and bone and coevally can minimise negative side effects which might be caused by systemically application. The PDLLA has been shown to be an appropriate coating for local release of various factors (Schmidmaier et al., 2001; Wildemann et al., 2004; Lucke et al., 2005; Pauly et al., 2009). In contrast to the current study, Hausman et al. reported a total absence of callus in the radiographs, a mainly uniform appearing fibrous tissue with few chondrocyte-like cells and minimal woven bone in the histology in the fractured and Fumagillin treated group. Furthermore, they reported a significant difference in the biomechanical properties between the fractured but untreated control and the fractured but treated group. The more pronounced differences in the biomechanical competence are explained by the regular healing pattern in the control. In the current study a delayed healing model was used for the control as well as for the treatment group and therefore we did not expected differences in the mechanical competence of the bones. In the study of Hausman et al. (2001) there were no remarks concerning the vascularity at the lesion side and therefore, the direct inhibiting effect of Fumagillin on angiogenesis was not proven. Other experimental models, that aim to investigate the vascularity of atrophic non-unions, have so far been created in rats and rabbits by severe mechanical manipulations (Triffit et al., 1993; Bronlow et al., 2002; Reed et al., 2003). Osteotomy in combination with destruction of the periosteum or endosteum and curettage of the intramedullary canal resulted in a significant reduction of blood vessels in the interfragmentary gap during the early post-operative phase and the subsequent development of non-unions. However, it is well accepted that the periost as well as the bone marrow provide various factors and cells, which are known to play a key role in bone repair. Therefore, in clinical situations, the protection of the periosteum is of high priority. With respect to this, we aimed to achieve the inhibition of neovascularisation after bone trauma using an alternative method.

An extreme model of disturbed vascularisation was used by Lu et al. (2007) by resection of the femoral artery in a mouse tibia fracture model. The ischemia resulted in an impaired bone healing with a decreased callus area including reduced callus and, in contrast to their expectations, reduced cartilage. In the present study however, the inhibition of angiogenesis caused a tendency towards a higher mineralised content with significant differences at day 42 . The cartilage area was slightly but not significantly enlarged.

The last could be seen in accordance with the well accepted effect of hypoxia on the differentiation of bone marrow stem cells in the chondrogenic lineage (Kanichai et al., 2008). Unlike the method applied by Lu et al. (2007) - which caused severe complications including toe, foot and hind limb necrosis as well as death - there were no indications of negative effects of Fumagillin on general health or gross perfusion of the lower leg in our study.

Further studies will be performed to investigate the healing over time using $\mu \mathrm{CT}$ to gain more information on the mineralisation of the callus, the callus formation and also the vascularisation at the later time points. Reed et al. established a rat atrophic non-union model using a $1 \mathrm{~mm}$ defect with cauterisation of the periost and endost (Reed et al., 2003). The investigation of the vascularisation revealed a reduced vessel number only at the early healing phase but not after 8 weeks. The group found similar results in a rabbit model (Brownlow et al., 2002). If the revascularisation is also impaired only in the early healing phase of the present atrophic non-union model, it must be evaluated in further studies. In addition, the callus remodelling will be investigated at the later time points with a special focus on osteoclast like cells. This important information is missing in the present study but is necessary for the description of the model.

In conclusion, we demonstrated that Fumagillin is a selective inhibitor of endothelial cell activity and reduces number of visible blood vessels in the chorioallantoic membrane of hen eggs. A reduction of early blood vessel number within the callus tissue can be achieved by local release. Our results indicated that angiogenesis inhibition has an impact on callus tissue formation and bone healing. We considered this model favourable to mimic the pathologic healing seen in human atrophic non-unions and to be suitable for efficacy testing of newly developed therapeutic strategies. For testing new drugs the induced non-union must be debrided and the nail with the Fumagillin coating removed. After this, a nail coated 
with a therapeutic drug can be inserted and the healing process monitored. Furthermore, this study can be used for continuing analysis of angiogenic and osteogenic factors.

The present study describes a clinically relevant rat model, which shows the formation of an atrophic nonunion after 84 days. The inhibition of angiogenesis resulted in a reduced vessel formation in the early healing phase accompanied with a smaller callus at the later healing phase. This model allows for the investigation of the pathology and also new treatment strategies.

\section{Acknowledgments}

We thank Dr. Christine Kratzel for the surgery as well as Marzena Princ, Alexander Schill, and Mario Thiele for their helpful technical assistance. We would like to thank Dr. Bettina Willie for proof reading of the manuscript. The work was supported by a grant from the Deutsche Forschungsgemeinschaft (DFG, SFB 760).

\section{References}

Ades EW, Candal FJ, Swerlick RA, George VG, Summers S, Bosse DC, Lawley TJ (1992): HMEC-1: establishment of an immortalised human microvascular endothelial cell line. J Invest Dermatol 99: 683-690.

Alex D, Leong EC, Zhang ZJ, Yan GT, Cheng SH, Leong CW, Li ZH, Lam KH, Chan SW, Lee SM (2010) Resveratrol derivative, trans-3,5,4'-Trimethoxystilbene, exerts antiangiogenic and vascular-disrupting effects in zebrafish through the downregulation of VEGFR2 and cell-cycle modulation. J Cell Biochem 109: 339-346.

Beamer B, Hettrich C, Lane J (2009) Vascular endothelial growth factor: an essential component of angiogenesis and fracture healing. HSS J 6: 85-94.

Belleri M, Ribatti D, Nicoli S, Cotelli F, Forti L, Vannini V, Stivala LA, Presta M (2005) Antiangiogenic and vascular-targeting activity of the microtubule-destabilizing trans-resveratrol derivative 3,5,4'-Trimethoxystilbene. Mol Pharmacol 67: 1451-1459.

Brinker MR, Bailey DE (1997) Fracture healing in tibia fractures with an associated vascular injury. J Trauma 42 : 11-19.

Brownlow HC, Reed A, Simpson AHRW (2002) The vascularity of atrophic non-unions. Injury 33: 145-150.

Calori GM, Phillips M, Jeetle S, Tagliabue L, Giannoudis PV (2008) Classification of non-union: need for a new scoring system? Injury 39: 59-63.

Carano RAD, Filvaroff EH (2003) Angiogenesis and bone repair. Drug Discov Today 8: 980-989.

Chen H, Zhou H, Wu G, Lou X (2004) Inhibitory effects of Artesunate on angiogenesis and on expressions of vascular endothelial growth factor and VEGF receptor KDR/flk-1. Pharmacology 71: 1-9.

Dai J, Rabie AB (2007): VEGF: an essential mediator of both angiogenesis and endochondral ossification. J Dent Res. 86: 937-950.

Deckers MM, van Bezooijen RL, van der Horst G, Hoogendam J, van Der Bent C, Papapoulos SE, Löwik
CW (2002): Bone morphogenetic proteins stimulate angiogenesis through osteoblast-derived vascular endothelial growth factor A. Endocrinology 143: 15451553.

Dell'Eva R, Pfeffer U, Vené R, Anfosso L, Forlani A, Albini A, Efferth T (2004) Inhibition of angiogenesis in vivo and growth of Kaposi's sarcoma xenograft tumors by the anti-malarial Artesunate. Biochem Pharmacol 68: 2359-2366.

Dickson KF, Katzman S, Paiement G (1995) The importance of the blood supply in the healing of tibial fractures. Contemp Orthop 30: 489-493.

Efferth T, Sauerbrey A, Olbrich A, Gebhard E, Rauch P, Weber HO, Hengstler JG, Halatsch ME (2003) Molecular modes of action of Artesunate in tumor cell lines. Mol Pharmacol 64: 382-394.

Einhorn TA (1995) Enhancement of fracture-healing. J Bone Joint Surg Am 77: 940-956.

Folkman J (2004) Endogenous angiogenesis inhibitors. APMIS 112: 496-507.

Folkman J (2007) Angiogenesis: an organizing principle for drug discovery? Nat Rev Drug Discov 6: 273-286.

Frémont L (2000) Biological effects of resveratrol. Life Sci 66: 663-673.

Giannoudis PV, Einhorn TA, Schmidmaier G, Marsh D (2008) The diamond concept - open questions. Injury 39: $5-8$.

Hausman MR, Schaffler MB, Majeska RJ (2001) Prevention of fracture healing in rats by an inhibitor of angiogenesis. Bone 29: 560-564.

Hazel SJ (2003) A novel early chorioallantoic membrane assay demonstrates quantitative and qualitative changes caused by antiangiogenic substances. J Lab Clin Med 141: 217-228.

Hietaniemi K, Peltonen J, Paavolainen P (1995) An experimental model for non-union in rats.Injury 26: 681686.

Hou L, Mori D, Takase Y, Meihua P, Kai K, Tokunaga O (2009) Fumagillin inhibits colorectal cancer growth and metastasis in mice: in vivo and in vitro study of antiangiogenesis. Pathol Int 59: 448-461.

Ingber D, Fujita T, Kishimoto S, Sudo K, Kanamaru T, Brem H, Folkman J (1990) Synthetic analogues of Fumagillin that inhibit angiogenesis and suppress tumour growth. Nature 348: 555-557.

Kanczler JM, Oreffo ROC (2008) Osteogenesis and angiogenesis: the potential for engineering bone. Eur Cell Mater 15: 100-114.

Kanichai M, Ferguson D, Prendergast PJ, Campbell VA (2008) Hypoxia promotes chondrogenesis in rat mesenchymal stem cells: a role for AKT and hypoxiainducible factor (HIF)-lalpha. J Cell Physiol 216: 708-715.

Keramaris NC, Calori GM, Nikolaou VS, Schemitsch EH, Giannoudis PV (2008) Fracture vascularity and bone healing: a systematic review of the role of VEGF. Injury 39: 45-57.

Klayman DL (1985) Qinghaosu (artemisinin): an antimalarial drug from China. Science 228: 1049-1055.

Kratzel C, Bergmann C, Duda G, Greiner S, Schmidmaier G, Wildemann B (2008) Characterization 
of a rat osteotomy model with impaired healing. BMC Musculoskelet Disord 9: 135.

Liu S, Widom J, Kemp CW, Crews CM, Clardy J (1998) Structure of human methionine aminopeptidase-2 complexed with Fumagillin. Science 282: 1324-1327.

Lu C, Miclau T, Hu D, Marcucio RS (2007) Ischemia leads to delayed union during fracture healing: a mouse model. J Orthop Res 25: 51-61.

Lucke M, Wildemann B, Sadoni S, Surke C, Schiller R, Stemberger A, Raschke M, Haas NP, Schmidmaier G (2005) Systemic versus local application of gentamicin in prophylaxis of implant-related osteomyelitis in a rat model. Bone 36: 770-778.

Pauly S, Luttosch F, Morawski M, Haas NP, Schmidmaier G, Wildemann B (2009) Simvastatin locally applied from a biodegradable coating of osteosynthetic implants improves fracture healing comparable to BMP-2 application. Bone 45: 505-511.

Reed AA, Joyner CJ, Brownlow HC, Simpson AH (2002): Human atrophic fracture non-unions are not avascular. J Orthop Res 20: 593-599.

Reed AAC, Joyner CJ, Isefuku S, Brownlow HC, Simpson AHRW (2003) Vascularity in a new model of atrophic nonunion. J Bone Joint Surg Br 85: 604-610.

Robey PG, Termine JD (1985): Human bone cells in vitro. Calcif Tissue Int 37: 453-460.

Schmidmaier G, Wildemann B, Stemberger A, Haas NP, Raschke M (2001) Biodegradable poly(D,L-lactide) coating of implants for continuous release of growth factors. J Biomed Mater Res 58: 449-455.

Schmidmaier G, Wildemann B, Melis B, Krummrey G, Einhorn TA, Haas NP, Raschke M (2004) Development and characterization of a standard closed tibial fracture model in the rat. Eur J Trauma 30: 35-42

Sin N, Meng L, Wang MQ, Wen JJ, Bornmann WG, Crews CM (1997) The anti-angiogenic agent Fumagillin covalently binds and inhibits the methionine aminopeptidase, MetAP-2. Proc Natl Acad Sci USA 94: 6099-6103.

Sivakumar B, Harry LE, Paleolog EM (2004) Modulating angiogenesis: more vs. less. JAMA 292: $972-$ 977.

Triffitt PD, Cieslak CA, Gregg PJ (1993) A quantitative study of the routes of blood flow to the tibial diaphysis after an osteotomy. J Orthop Res 11: 49-57.

Tzioupis C, Giannoudis PV (2007) Prevalence of longbone non-unions. Injury 38: 3-9.

Wang J, Lou P, Henkin J (2000) Selective inhibition of endothelial cell proliferation by fumagillin is not due to differential expression of methionine aminopeptidases. J Cell Biochem 77: 465-473.

Weber BG (1982) Klassifizierung der Pseudarthrosen nach morphologischen und klinischen Gesichtspunkten (Classification of pseudo-arthroses from a morphological and clinical point of view). Z Orthop 120: 528-531

Wildemann B, Bamdad P, Holmer C, Haas NP, Raschke M, Schmidmaier G (2004) Local delivery of growth factors from coated titanium plates increases osteotomy healing in rats. Bone 34: 862-868.
Ye C, Sweeny D, Sukbuntherng J, Zhang Q, Tan W, Wong S, Madan A, Ogilvie B, Parkinson A, Antonian L (2006) Distribution, metabolism, and excretion of the anti-angiogenic compound SU5416. Toxicol In Vitro 20: 154-162.

Zhang Y, Yeh JR, Mara A, Ju R, Hines JF, Cirone P, Griesbach HL, Schneider I, Slusarski DC, Holley SA, Crews CM (2006) A chemical and genetic approach to the mode of action of Fumagillin. Chem Biol 13: 1001-1009.

Zhou H, Wang W, Wu G, Lee J, Li A (2007) Artesunate inhibits angiogenesis and downregulates vascular endothelial growth factor expression in chronic myeloid leukemia K562 cells Vascul Pharmacol 47: 131-138.

\section{Discussion with Reviewers}

Reviewer I: Can the authors describe the evaluation of the vessel formation? Which parameters were scored? Sizes, amount, arborisation?

Authors: This is an important question and we are sorry that we are not able to answer it satisfactorily. The vessels were scored by three independent observers but no quantitative analysis was done. Due to difficulties in the quantitative evaluation of vessel number, length and diameter we decided to perform only the score analysis. But for the identification of the angiogenesis inhibitor used in the in vivo model the evaluation method was sufficient.

Reviewer I: Did the authors perform antibody retrieval? Authors: We did not perform an antibody retrieval experiment. The identification of the vessels was based on the smooth muscle actin-staining and the morphology and therefore only vessels were counted.

Reviewer I: Apart from the reduced metabolic activity and the reduced vessel density, could the authors observe changes in vascular organization in vivo?

Authors: Based on our methods we cannot give any information regarding changes in the vascular organization. A three dimensional analysis of the vascularity (e.g., by the microfilling of vessels and subsequent $\mu \mathrm{CT}$ imaging) would be necessary, but this has not been done in the present study.

Reviewer I: What is the underlying effect of the antiangiogen activity?

Authors: It is known that fumagillin inhibits neovascularisation by endothelial cell cycle arrest in the late G1 phase. For the fumagillin analogue TNP-470 it was shown that it inhibits the gene product phosphorylation as well as the cyclin dependent kinase activation and expression of cyclins E and A (Abe et al., 1994, additional reference). Binding of Fumagillin to the methionineaminopeptidase type II results in the block of the G1 progression (Sin et al., 1997, text reference) which leads more downstream to a block of the noncanonical Wntsignalling pathway (Zhang et al., 2006, text reference). 
Reviewer I: Although there was no effect on the osteoblast metabolic activity in vitro, do the authors expect side effects on osteoblast or hMSC differentiation?

Authors: As described in the introduction, the study by Zhang et al. (2006) showed a blocking of the Wntsignalling pathway due to the fumagillin application. In both cell types, osteoblast like cells and MSC, the Wnt-signalling pathway is essential for the cell fate and differentiation and a disturbance of the signalling pathway results in altered bone mass. Therefore, we cannot exclude that there might be a side effect of the applied Fumagillin also directly on bone formation. But in the osteoblast experiments, we saw no significant effect on this cell type.

Reviewer II: Which potential therapies could be testing using this model, given that the angiogenesis inhibitor will still be present for the entire experiment and thus also inhibit any treatment effects?

Authors: This model can be used to analyse treatment options because in the clinical situation the treatment of the non-union includes a revision operation with debridement and optionally the removal of the implant. In this model this means also the removal of the drug carrier and the fumagillin. For the treatment an implant coated with the therapeutically drug can then be used.

Reviewer II: Could a short burst of an angiogenesis inhibitor in the critical first week be as useful as continuous administration?

Authors: We are now working on the modification of the drug release that will than allow a better adjustment of the drug activity. If an inhibition in the first week will be sufficient, however, that must be tested.

Reviewer II: In this interesting model, the authors show blood vessel reduction in the Fumagillin group at day 5 and 10. Do the authors know the extent of blood vessel numbers at day 42 and 84 - thus was there a total reduction in blood vessels in the defect region at these time points or was blood vessel angiogenesis reduced at the early time points and increased over time in the PDLLA and Fumagillin treated groups?

Authors: Unfortunately, we have only analysed the blood vessels at day 5 and 10. At the later time points the specimen were plastic embedded and the immunohistochemical staining of smooth muscle actin did not work properly.
Therefore, we cannot give information regarding the later time points.

Reviewer II: Could the authors comment on whether there was any increase or reduction in the remodelling of the callus (such as number of osteoclasts) in the later stages of the bone repair in this model?

Authors: We agree that the later time points will give important information regarding the remodelling. The specimens of the later time points, however, were plastic embedded and we tried a TRAP stain but the staining was not reproducible. Based on the Movat Pentachrome staining osteoclasts were identifiable, but a quantitative analysis was not possible.

Reviewer III: In Fig. 4, the authors show blood vessel reduction in the Fumagillin group at day 5 and 10. No data is presented for blood vessel numbers at day 42 and 84 . Was there a total reduction in blood vessels in the defect region at these time points or was blood vessel angiogenesis reduced at the early time points and increased over time in the PDLLA and Fumagillin treated groups? Was there any increase/reduction in the remodelling of the callus such as number of osteoclasts etc in the later stages of the bone repair?

Authors: This is a critical point of the study and unfortunately we cannot give any information regarding the vessel number at later time points. We tried to stain the plastic embedded tissues with the $\alpha$-smooth muscle actin antibody but the staining result was not very good and reproducible. Therefore, we have no analysis of vessel formation for the later time points. In addition we tried to stain the osteoclasts with the TRAP stain but this also did not work properly on plastic embedded bone tissue.

\section{Additional Reference}

Abe J, Zhou W, Takuwa N, Taguchi J, Kurokawa K, Kumada M, Takuwa Y (1994) A fumagillin derivative angiogenesis inhibitor, AGM-1470, inhibits activation of cyclin-dependent kinases and phosphorylation of retinoblastoma gene product but not protein tyrosyl phosphorylation or protooncogene expression in vascular endothelial cells. Cancer Res 54: 3407-3412. 